\title{
Control of superconductivity with a single ferromagnetic layer in niobium/erbium bilayers
}

\author{
N. Satchell, ${ }^{1,2}$ J. D. S. Witt, ${ }^{1}$ M. G. Flokstra, ${ }^{3}$ S. L. Lee, ${ }^{3}$ \\ J. F. K. Cooper, ${ }^{2}$ C. J. Kinane, ${ }^{2}$ S. Langridge, ${ }^{2}$ and G. Burnell ${ }^{1, \text { * }}$ \\ ${ }^{1}$ School of Physics and Astronomy, \\ University of Leeds, Leeds, LS2 9JT, United Kingdom \\ ${ }^{2}$ ISIS Neutron and Muon Source, STFC Rutherford Appleton Laboratory, \\ Chilton, Didcot, Oxon, OX11 0QX, United Kingdom \\ ${ }^{3}$ School of Physics and Astronomy, SUPA, \\ University of St Andrews, St Andrews KY16 9SS, United Kingdom
}

(Dated: April 8, 2017)

\begin{abstract}
Superconducting spintronics in hybrid superconductor-ferromagnet $(\mathrm{S}-\mathrm{F})$ heterostructures provides an exciting potential new class of device. The prototypical super-spintronic device is the superconducting spin-valve, where the critical temperature, $T_{c}$, of the S-layer can be controlled by the relative orientation of two (or more) F-layers. Here, we show that such control is also possible in a simple $\mathrm{S} / \mathrm{F}$ bilayer. Using field history to set the remanent magnetic state of a thin Er layer, we demonstrate for a $\mathrm{Nb} /$ Er bilayer a high level of control of both $T_{c}$ and the shape of the resistive transition, $\mathrm{R}(\mathrm{T})$, to zero resistance. We are able to model the origin of the remanent magnetization, treating it as an increase in the effective exchange field of the ferromagnet and link this, using conventional S-F theory, to the suppression of $T_{c}$. We observe stepped features in the $\mathrm{R}(\mathrm{T})$ which we argue is due to a fundamental interaction of superconductivity with inhomogeneous ferromagnetism, a phenomena currently lacking theoretical description.
\end{abstract}

* g.burnell@leeds.ac.uk 


\section{INTRODUCTION}

While traditionally considered competing phenomena, when artificially juxtaposed, there is a wealth of physics at the interface between superconductors $(\mathrm{S})$ and ferromagnets $(\mathrm{F})$. Taking advantage of the competition between order parameters has lead to advances in the emerging field of super-spintronics [1]. By placing an inhomogeneous magnetic texture at the $\mathrm{S}-\mathrm{F}$ interface, it is possible to create the so-called long ranged triplet component (LRTC) or finite spin Cooper pair. Unlike the singlet Cooper pair, the LRTC is not dephased by the exchange field and can therefore penetrate further into a proximitised F-layer. This opens the exciting possibility of performing spintronic logic operations on a dissipationless spin current [2]. Additionally, several breakthroughs in complex S-F heterostructures show promise as potential cryogenic memory elements. In such a scheme, information could be stored by the state of the system (superconducting or normal) [3, 4] or the ground-state phase difference between two S-layers in an S/F/S Josephson junction [5].

The prototypical super-spintronic device is the superconducting spin valve. In this device, control of the magnetic state of the two F-layers in an $\mathrm{S} / \mathrm{F} / \mathrm{F}$ or $\mathrm{F} / \mathrm{S} / \mathrm{F}$ heterostructure can be used to tune the generation of the LRTC [10 15]. The generation of the LRTC opens an additional conduction channel for Cooper pairs, resulting in the lowering of $T_{c}$ [16]. In our previous work we found this suppression of $T_{c}$ to be of the order $10-20 \mathrm{mK}$ in a $3 d$ ferromagnet/niobium device [13], although other works have increased this effect; to 130-140 mK by carefully engineering both the superconducting layer and S-F interface [12, 15], and to over $1 \mathrm{~K}$ by both introducing a half-metal as the bottom F-layer and changing the applied field orientation from an in-plane rotation to an in-plane to out-of-plane rotation [17, 18]. The manipulation of the F-layers in the superconducting spin valve requires careful engineering of the heterostructure and the rotation of the sample in an applied magnetic field. Under an in-plane field rotation it is possible to introduce experimental artefacts due to: vortex flow (if too high a current is applied, induced voltage from vortex flow will dominate the transport signal); non-uniformity of field (if the sample is not aligned correctly the out-of-plane field component will vary under rotation - modifying $T_{c}$ ); and temperature (a temperature gradient or local source of heating inside a cryostat is an important consideration when the sample is moving during measurement). Any of these can introduce a signal with the same periodicity as the signature of LRTC generation. A recent theoretical work considered that 
there exists a "half-select" problem in the multilayer spin valve approach, which may be negated in a simplified device [19].

In this work we describe a simplified S-F hybrid system, where the superconductivity can be controlled by a single adjacent F-layer. The system only requires the ability to apply an external field in one direction (without the need for sample rotation) and we perform all our measurements in zero applied field, two distinct advantages over the superconducting spin valve. This is achieved by coupling a superconducting Nb layer to rare-earth ferromagnetic Er, which has a large number of metastable magnetic phases accessible with temperature or applied field. Previous work on holmium and dysprosium demonstrate the important role rare-earth ferromagnets will play in the implementation of superconducting spintronics in Josephson type devices [20, 21] and devices based on the control of $T_{c}$ [22, 23]. For example, Gu et al. demonstrated that an antiferromagnetic to ferromagnetic transition in Ho resulted in modification of the $T_{c}$ of an adjacent $\mathrm{Nb}$ layer of over $100 \mathrm{mK}$, however the exact mechanism involved in the $T_{c}$ shift was not established [22]. This work was later expanded by producing trilayer samples of $\mathrm{Ho} / \mathrm{Nb} / \mathrm{Ho}$ and $\mathrm{Dy} / \mathrm{Nb} / \mathrm{Dy}$ in which a spin valve like effect of $400 \mathrm{mK}$ was discovered [23]. These works established the ability to control $T_{c}$ with the ferromagnetic texture in rare-earth ferromagnets, however lacked the theoretical description and the additional modification to the shape of the $\mathrm{R}(\mathrm{T})$ transition reported in this manuscript.

Er is a trivalent rare-earth metal $(Z=68)$, with highly localised 4-f electrons and a hexagonal close packed (hcp) crystal structure. Competition between the RKKY indirect exchange interaction and the crystalline anisotropy, creates a rich magnetic phase diagram making this material ideal for the exploration of S-F proximity effects [24 31]. Below the hightemperature paramagnetic phase $(\approx 85 \mathrm{~K})$, Er first gains a sinusoidal, $c$-axis modulated $(\mathrm{CAM})$ anti-ferromagnetic phase. As the temperature is lowered, the magnetic wave vector of the CAM expands until $\approx 52 \mathrm{~K}$. Below this temperature Er enters an 'intermediate' phase where the in-plane moments begin to order creating what has been referred to by Cowley et al. as an anti-ferromagnetic "wobbling cycloid" [27. The magnetic cycloid repeat distance increases with decreasing temperature, through a number of stable commensurate phases, to 8 atomic layers. These states exhibit a ferrimagnetic moment. Finally, below $18 \mathrm{~K}$ a conical $c$-axis ferromagnetic phase is formed. We have been able to confirm that many of the magnetic states of bulk Er are reproducible in sputter deposited epitaxial thin films, and 
that these magnetic states can be controlled with either temperature or applied magnetic field [32, 33].

\section{METHODS}

The films were deposited using DC sputtering in a system with substrate heaters mounted above each sample slot. At the highest temperature, the base pressure of the system is $\approx 10^{-7}$ mbar. This pressure improves as the system temperature is lowered. The samples were grown on $0.65 \mathrm{~mm}$ thick $c$-plane $\mathrm{Al}_{2} \mathrm{O}_{3}$ substrates. The $\mathrm{Nb}$ was deposited at a nominal temperature of $700^{\circ} \mathrm{C}$, after which the system was cooled to $500^{\circ} \mathrm{C}$, a final thin $\mathrm{Nb}$ interface layer was deposited at this temperature, followed directly by the Er and then a $5 \mathrm{~nm}$-thick Lu capping layer. Growth was performed at a typical Ar flow of $55 \mathrm{sccm}$ resulting in Ar pressure of 2-3 $\mu$ bar, at a substrate-sample distance of $75 \mathrm{~mm}$, and at a typical growth rate of $0.1 \mathrm{~nm} \mathrm{~s}^{-1}$. Growth rates were calibrated by fitting to Keissig fringes obtained on single layer samples by X-ray reflectometry. The $\mathrm{Nb}$ was grown first as it has been shown to be an effective buffer layer for the growth of rare-earth metals and stops the Er layer reacting with oxygen in the substrate [34]. The Nb/Er interface is known to be sharp due to the lack of alloying and intermixing between $\mathrm{Nb}$ and $\mathrm{Er}$ [35]. The Er grows epitaxially on the most densely packed $\mathrm{Nb}$ (110) plane, in the Nishiyama-Wasserman orientation. The in-plane axis

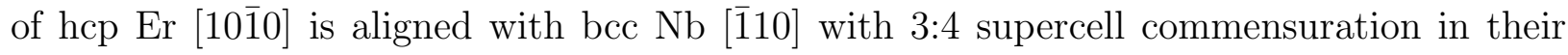
nearest-neighbour distances along these axes [34]. Lu was chosen for the capping layer as it lattice matches well with Er (preventing additional strain being introduced), and unlike some traditional capping metals, such as $\mathrm{Au}$, it can be deposited as a continuous layer at high temperatures [32].

Magnetization loops and remanent magnetization, $M_{r}$, were measured using a 6 T Quantum Designs SQUID-VSM magnetometer at $10 \mathrm{~K}$. Electrical transport measurements were performed on sheet films using a conventional four point probe measurement configuration

and employing two continuous flow ${ }^{4} \mathrm{He}$ cryostats, with maximum fields of $3 \mathrm{~T}$ and $8 \mathrm{~T}$. The field histories were only applied when the sample was in the normal state (to prevent flux trapping). The resistance as a function of temperature $(\mathrm{R}(\mathrm{T}))$ of the sample, from which $T_{c}$ is obtained, was always measured at zero applied field. Temperature sweeps, both cooling and warming, were recorded to check for temperature hysteresis in the measurements. The 
temperature hysteresis (observable in FIG. 2) does not account for the observed $T_{c}$ shift in FIG. 3.

\section{RESULTS}

\section{A. Magnetic Characterization}

The magnetization versus field data, along with minor loops, for the $25 \mathrm{~nm}$-thick Er bilayer sample at $10 \mathrm{~K}$ are shown as the inset in FIG. 1 (a). The red squares show the initial magnetization and full magnetic hysteresis behaviour for applied magnetic fields up to $60 \mathrm{kOe}$. The solid lines are a series of minor loops, from which information about the $M_{r}$ of the sample can be obtained. The $M_{r}$ as a function of initial field data are collated in FIG. 1 (a).

It is evident from FIG. 1 (a) that for low initial fields, there is little change to the remanent state of the Er. This indicates that, in this range, the stabilisation of the spiral magnetic structure in the Er-due the RKKY interaction - is robust against perturbation by the externally applied magnetic fields. The large increase in $M_{r}$ for initial fields of about $10 \mathrm{kOe}$ is evidence that, for initial field values greater than this, the Er does not reenter the same magnetic phase upon relaxation of the field. This is consistent with previous characterization work which shows that at approximately $25 \mathrm{kOe}$ there is a phase transition, for an in-plane applied field, into a distorted spiral phase, known as the 'fan' or 'canted-fan' state [31, 32]. Possible origins of the increased remanence are shown schematically in FIG. 1 (b) and discussed further in section IV A.

\section{B. Electrical Transport}

FIG. 2 shows the resistance as a function of temperature for the $25 \mathrm{~nm}$-thick Er bilayer sample, always measured in zero applied field. The data show the onset of superconductivity as the temperature is decreased for the virgin state (triangles) and after the application of an $80 \mathrm{kOe}$ applied magnetic field (squares). In the inset of FIG. 2 the evolution of R(T) as a function of the initial applied magnetic field can be seen. Resistance as a function of temperature was always measured in zero applied magnetic field and $T_{c}$ was defined as $50 \%$ of the normal state resistance. $\Delta T_{c}$ is calculated as the difference between $T_{c}$ of the virgin 

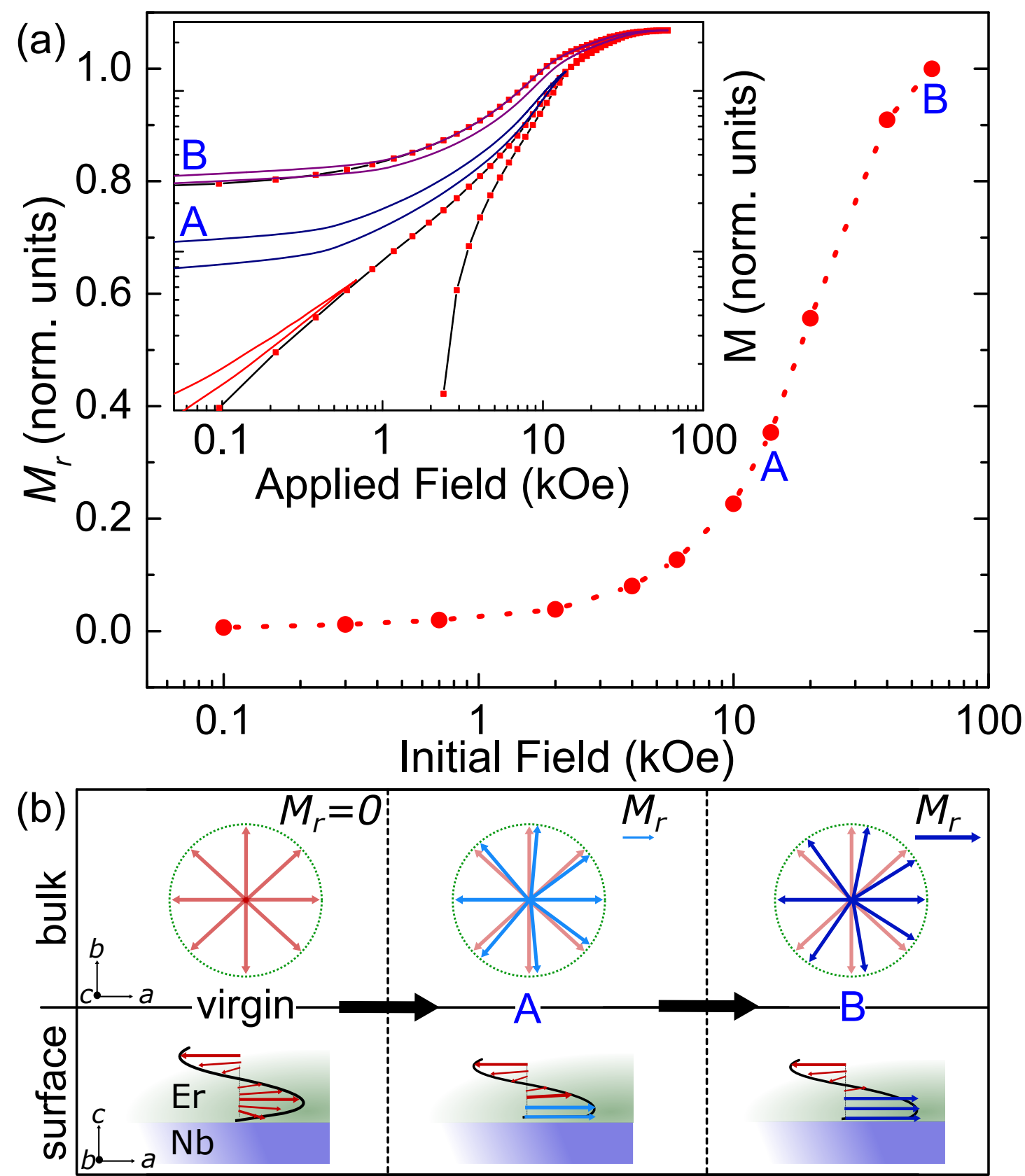

FIG. 1. (a) The normalised remanent magnetization, $M_{r}$, as a function of initial field. Inset: Positive quadrant of the magnetic hysteresis loop (squares) and exemplar minor loops (solid lines) of the bilayer at $10 \mathrm{~K}$. The data are displayed on a log-log plot for increased clarity. (b) The two possible mechanisms to account for $M_{r}$. The bulk mechanism shows a canting of the spiral into the direction of the applied field. The surface mechanism shows an interface effect, where only the surface moments remain aligned in the direction of the applied field. 


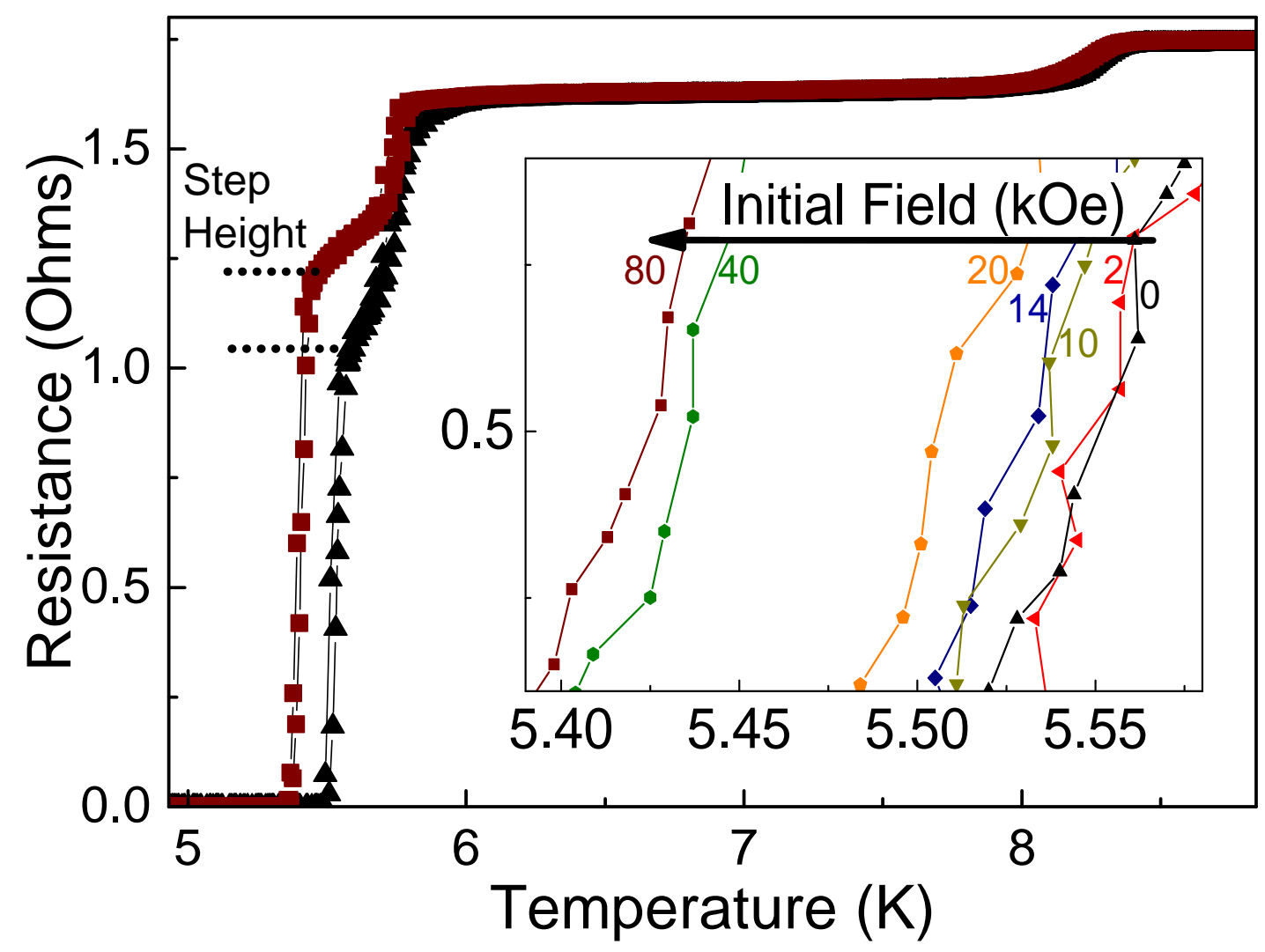

FIG. 2. Resistance as a function of temperature $(\mathrm{R}(\mathrm{T}))$ for the virgin magnetic state (triangles) and post $80 \mathrm{kOe}$ field saturation (squares). Both warming and cooling data are included and the changing step heights marked. Inset: Exemplar cooling curves demonstrating the evolution of $\mathrm{R}(\mathrm{T})$ with initial field.

state (triangles in FIG. 2) and $T_{c}$ after the application and removal of a magnetic field. The $\Delta T_{c}$ data for all of the initial applied magnetic field values are collated in FIG. 3 .

In FIG. 3 it is immediately clear that there is a strong link between the $M_{r}$ of the Er and the $T_{c}$ of the superconductor. This correlation, between the properties of Er and Nb, show that both the $T_{c}$ of the $\mathrm{Nb}$ and the magnetic state of the Er are strongly dependent upon the field history of the sample. It also shows that there is a strong coupling between the superconducting and magnetic layers. The largest change to $\Delta T_{c}$ comes between $20-30 \mathrm{kOe}$, which, as mentioned above, is also the field value where the Er state changes magnetic phase. After the application of the largest field possible in our system, $80 \mathrm{kOe}$, the $T_{c}$ of the $\mathrm{Nb}$ was suppressed by approximately $140 \mathrm{mK}$, which is the largest value reported for such a system. The metastable magnetic state obtained by applying and removing and initial field, 


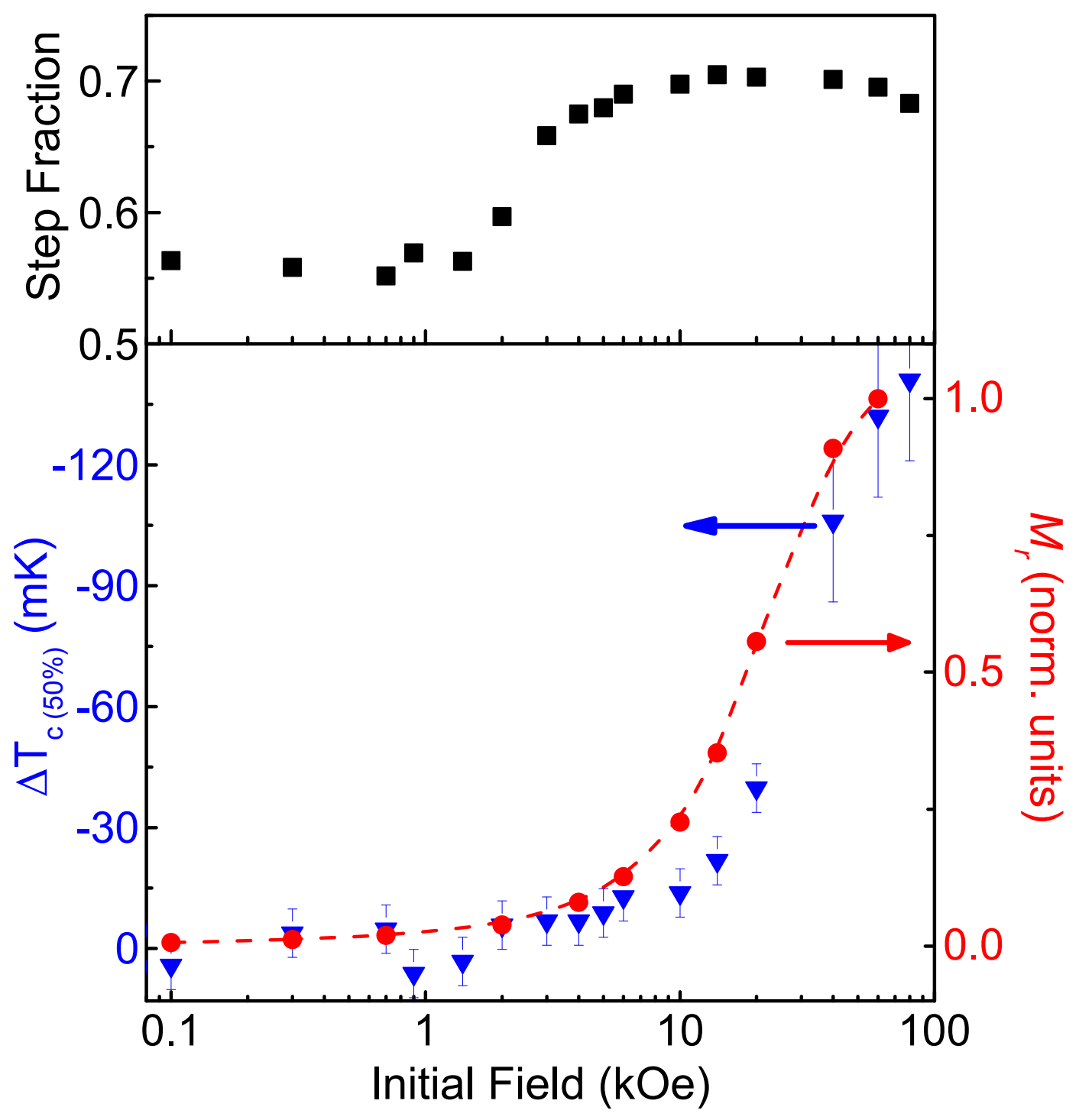

FIG. 3. Top panel: The fractional step height change with initial field. Bottom panel: The normalised remanent magnetization, $M_{r}$, (circles - reproduced from FIG 1) and the shift in superconducting critical temperature from the as cooled state, $\Delta T_{c}$, (triangles) as a function of initial applied magnetic field.

is robust against temperature changes in the measured range 5-10 K. The system can be effectively reset to the virgin state by warming through the Curie temperature.

One additional point to note is the step-like features that are present in FIG. 2, and the fact that these steps also change with field history. The height of the stepped feature in the transition is marked on FIG. 2. A step fraction is defined as the height of the step relative 
to the normal state resistance (at $10 \mathrm{~K}$ ). The collated step fraction is plotted in the upper panel of FIG. 3 and is discussed further in section $\mathrm{V}$.

\section{MODELLING}

\section{A. Modelling of $M_{r}$ : An Effective $E_{e x}$}

Having established that the suppression observed in $T_{c}$ is linked to the increased $M_{r}$, we now consider the local magnetic state of the Er film and propose two physical interpretations for the origin of $M_{r}$ in Er. The first being a 'bulk' modification of the spiral and the second a localised spin alignment at the Er surface.

As we have shown in our previous work, even in the thin film, Er has a highly complicated phase diagram [32, 33]. Through a combination of temperature and field, the Er can be placed in a number of metastable magnetic states. For an in-plane field, between 20 and $30 \mathrm{kOe}$ Er undergoes a transition from the conical to a 'fan' magnetic state, canted into the direction of applied field. Subsequent removal of the applied field causes the Er to re-enter the conical state, however we argue the cone has now been modified and is canting in the direction of the external applied field, increasing $M_{r}$. This canted conical state is shown schematically as the top mechanism in FIG. 1 (b).

It is well known that finite-size effects play an important part for thin film rare-earths [36]. The long-range nature of the RKKY interaction (up to 6th nearest neighbour) means that the reduced atomic coordination at the surfaces makes the spiral ends less robust against external perturbation, which clearly becomes more of an influencing factor for thinner films with a lower volume to surface area ratio [33]. It is, therefore, possible that, under the influence of an externally applied magnetic field, the spiral unwinds more readily at the surfaces and, being unable to overcome the energy barrier to reform the spiral, remains locally in the direction of the applied field. This is shown schematically as the bottom mechanism in FIG. 1 (b). We calculate from the known thickness, saturation magnetization and expected moment per atom that $0.65 \mathrm{~nm}$ (or just over two atoms) remaining aligned at the interface would account for the observed $M_{r}$ (see SI FIG 1).

The 'bulk' canted magnetic phase which leads to a net magnetization could be described by an effective exchange field if the coherence length inside the Er is (much) longer than the 
magnetic repeat unit of the helix, which is about $4 \mathrm{~nm}$. On the other hand, the contribution of the surface moments to the total proximity effect is only considerable if the coherence length is short, comparable to the $0.65 \mathrm{~nm}$ effective Er thickness corresponding to aligned surface moments. The two mechanisms thus correspond to very different length scales of the coherence length inside the Er layer.

Resistance measurements on Er/Nb bilayers with various Er layer thicknesses suggest an approximate coherence length of $10 \mathrm{~nm}$ (see SI FIG 2) and in a related Ho system the coherence length was estimated to be $30 \mathrm{~nm}$ [23]. This distance is far greater than $0.65 \mathrm{~nm}$ and is long enough to allow the Cooper pair to experience multiple helicies. It is, therefore, most likely that the Er undergoes the 'bulk' transition to a new canted magnetic phase, which is retained at zero field, and that this is the origin of the suppression in $T_{c}$.

\section{B. Modelling of $\Delta T_{c}$ vs. $M_{r}$}

To investigate the effect of an increased bulk remanent magnetisation, we model it as an effective exchange field $\left(J_{z}\right)$ in the $\mathrm{F}$ layer, which we can then link directly to the suppression in $T_{c}$.

Using the quasiclassical theory for superconductivity in the dirty limit (electronic mean free path much shorter than the phase coherence length), we calculate the critical temperature of a Er/ $\mathrm{Nb}$ bilayer as function of an effective exchange field inside the Er. We take the $\mathrm{x}$-axis normal to the metallic layers and assume translational invariance in the y,z plane. The Usadel equation for $s$-wave superconductivity then takes the form $i \hbar D \partial_{x}\left(\check{g} \partial_{x} \check{g}\right)=[\check{H}, \check{g}]$ with $\check{g}$ the $4 \times 4$ matrix Green function in the Nambu spin space, $\hbar$ the reduced Planck constant and $D$ the diffusion constant. For collinear exchange fields the Hamiltonian can be described by $\check{H}=i \hbar \omega_{n}\left(\tau_{3} \otimes \sigma_{0}\right)+\check{\Delta}-J_{z} \tau_{0} \otimes \sigma_{3}$ (see e.g. [37]) with $J_{z}$ the exchange field directed along the z-axis and $\omega_{n}$ the Matsubara frequencies defined by $\hbar \omega_{n}=\pi k_{B} T(2 n+1)$ with $k_{B}$ the Boltzmann constant, $n$ integer, and the maximum allowed frequency given by the Debye frequency. $\mathrm{x}-\mathrm{y}-\mathrm{z}$ is defined such that $J_{z}$ points along the direction of the net

moment of the Er. Furthermore, $\sigma_{i}$ and $\tau_{i}$ are the Pauli matrices of respectively the spin space and Nambu space. The matrix Green function and $\check{\Delta}$ have the following non-zero 


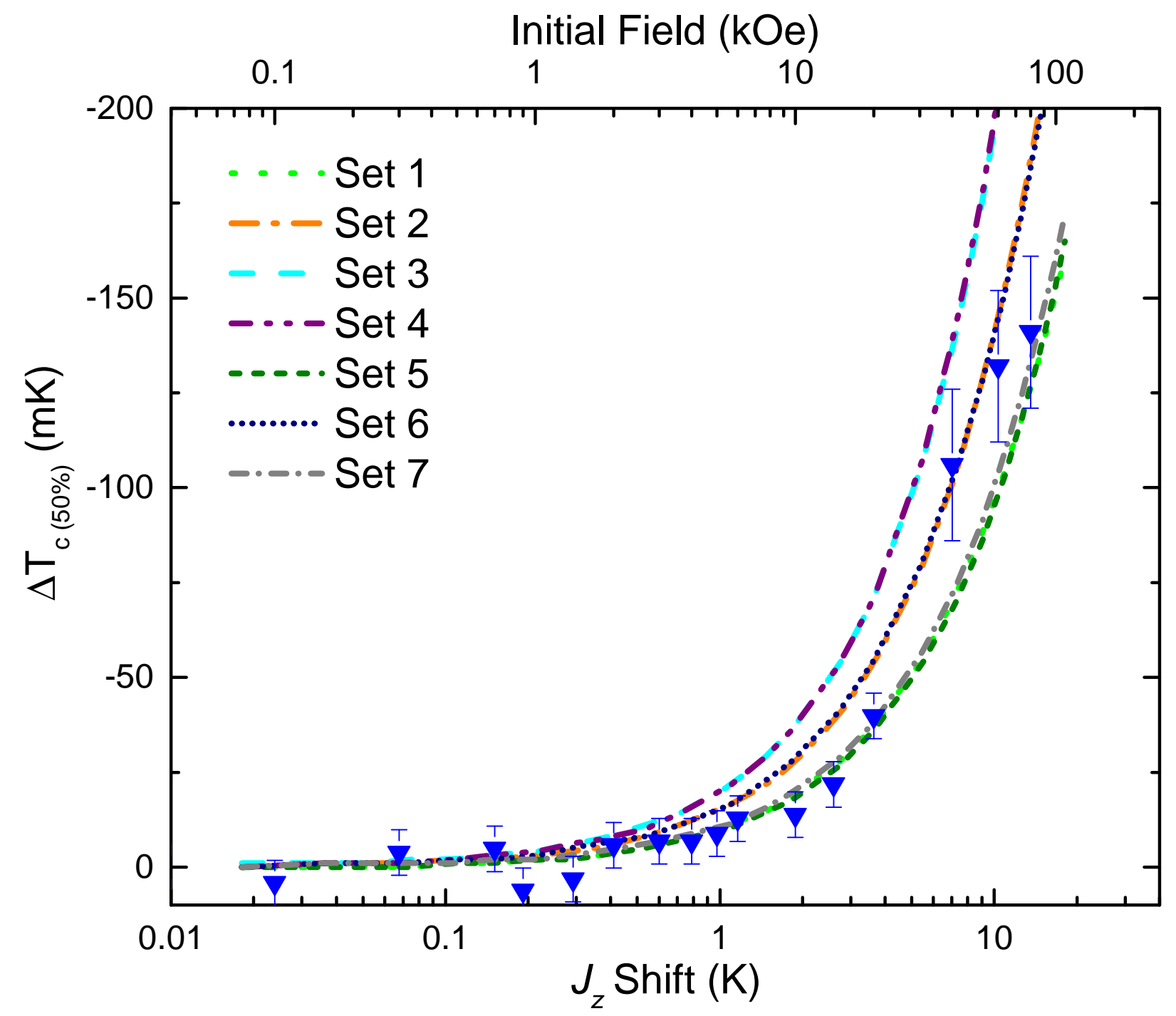

FIG. 4. The calculated suppression of $T_{c}$ with an effective shift to the exchange field $\left(J_{z}\right)$. Also plotted are the $\Delta T_{c}$ data from FIG. 3. The parameter 'sets' used in the calculation are defined in the text and listed in the SI Table 1.

elements:

$$
\check{g}=\left(\begin{array}{cccc}
G_{\uparrow \uparrow} & 0 & 0 & F_{\uparrow \downarrow} \\
0 & G_{\downarrow \downarrow} & F_{\downarrow \uparrow} & 0 \\
0 & \bar{F}_{\uparrow \downarrow} & \bar{G}_{\uparrow \uparrow} & 0 \\
\bar{F}_{\downarrow \uparrow} & 0 & 0 & \bar{G}_{\downarrow \downarrow}
\end{array}\right), \check{\Delta}=\left(\begin{array}{cccc}
0 & 0 & 0 & -\Delta \\
0 & 0 & \Delta & 0 \\
0 & -\Delta^{*} & 0 & 0 \\
\Delta^{*} & 0 & 0 & 0
\end{array}\right)
$$

where $G$ and $F$ are the quasiclassical normal and anomalous Green functions respectively, both functions of $\left(x, \omega_{n}\right)$, and $\Delta(x)$ is the order parameter. The matrix Green function satisfies the normalization condition $\check{g}^{2}=\check{1}$ and the order parameter must be solved self- 
consistently satisfying the gap equation:

$$
i \Delta(\mathbf{R})=\frac{\pi k_{B} T}{\ln \left(\frac{T}{T_{c 0}}\right)+\sum_{n}\left(\frac{1}{|2 n+1|}\right)} \sum_{\omega_{n}} F_{\uparrow \downarrow}\left(\mathbf{R}, \omega_{n}\right)
$$

with $T_{c 0}$ the bulk critical temperature. We use the interface boundary conditions as formulated by Nazarov 38] which for the interface between two materials with labels $l, r$ for the layer on the left and right side of the interface respectively can be written as: $\sigma_{l} \check{g}_{l} \partial_{x} \check{g}_{l}=\sigma_{r} \check{g}_{r} \partial_{x} \check{g}_{r}$ and $\sigma_{l} \check{g}_{l} \partial_{x} \check{g}_{l}=\frac{2}{R_{b}} \frac{\left[\check{g}_{l} \check{g}_{r}\right]}{4+\Gamma\left(\check{g}_{l} \check{g}_{r}+\check{g}_{r} \check{g}_{l}-2\right)}$, with $\sigma_{i}$ the conductivity of layer $i$, $0 \leq \Gamma \leq 1$ the interface transparency and $R_{b}$ the interface resistance times the interface area $\left(\Omega \mathrm{m}^{2}\right)$.

The material parameters used for the $\mathrm{Nb}$ layer are $\xi_{s}=\sqrt{\hbar D_{s} /\left(2 \pi k_{B} T_{\mathrm{c} 0}\right)}=7.9 \mathrm{~nm}$, $T_{c 0}=8.4 \mathrm{~K}$ and $\rho_{s}=15.2 \mu \Omega \mathrm{cm}$. Since the value of $J_{z}$ is unknown we explored various combinations of $\xi_{f}=\sqrt{\hbar D_{f} / J_{z}}, J_{z}$ and $R_{b}$ chosen such that the $T_{c}$ of the bilayer corresponds to the experimental value of $5.5 \mathrm{~K}$. For all calculations $\Gamma=1$. For each material combination $T_{c}$ was calculated as a function of a shift in $J_{z}$ (a shift of zero corresponding to the $T_{c}$ of 5.5 $\mathrm{K})$.

The results of the modelling are presented in FIG. 4 along with the experimental data. When taken with the thickness dependence, SI FIG. 2, it is parameter set 2 and 5 which show closest agreement to the experimental data, although all parameter sets considered qualitatively reproduce the experimental data. These two parameter sets give the same value of interface resistance, but were considered with different initial values of $J_{z}$, set 2 corresponding to the lowest temperature $(\approx 22 \mathrm{~K})$ conical ferromagnetic transition, and set 5 the transition from the antiferromagnetic to ferromagnetic "wobbling cycloid" intermediate state $(\approx 55 \mathrm{~K})$, both of which have been confirmed in our thin films [32]. From either starting point, the analysis shows that the observed $140 \mathrm{mK} T_{c}$ shift corresponds to a $5-10 \mathrm{~K}$ shift in $J_{z}\left(7.5-15 \times 10^{-20} \mathrm{meV}\right)$.

\section{DISCUSSION}

Rare earth ferromagnets, such as Er, offer a plethora of magnetic configurations in which a Cooper pair (coherent with a neighbouring proximity coupled superconductor) can experience magnetic disorder. As a conical ferromagnet, Er is a theoretically ideal system 
in which to generate and study proximity effects induced by an additional LRTC [39]. In this work it is expected that all $\mathrm{R}(\mathrm{T})$ measurements were performed when the Er was in a disordered magnetic state. It is therefore not possible to directly attribute LRTC generation to the observed $\Delta T_{c}$. In comparison to the superconducting spin valve, which has a clean LRTC on/off mechanism (as magnetic inhomogeneity is carefully engineered from otherwise homogeneous magnetic layers), our proposed origin of $M_{r}$ cannot provide such a switching mechanism. The canting of the magnetic state into the direction of applied field is unlikely to significantly change the conversion efficiency of singlet Cooper pairs into the LRTC. In the second proposed mechanism, spins at the surface remain aligned with the applied field, and could create a homogeneous interface layer. From the spin valve experimental argument we would expect this to result in a decrease in LRTC generation and therefore an increase in $T_{c}$. This does not agree with the experimental observation in this work.

Given the size of this $T_{c}$ effect is generally larger than that reported for spin-valves (and the number of reported cases showing an effect opposite to the spin-valve effect, where the disordered magnetic state results in a higher measured $T_{c}$ [22, 40,42]), we urge caution for the interpretation of $T_{c}$ measurements alone as evidence for the presence of the LRTC in S-F systems.

With the modelling we have shown that the reported change in $T_{c}$ can be described within the conventional S-F proximity theory by considering the increasing remanence of the Er as a shift in the effective exchange field. This increase in exchange field modifies the proximity effect, suppressing the $T_{c}$ of the bilayer. An effective shift in $J_{z}$ of $5-10 \mathrm{~K}$ accounts for the observed changes in $T_{c}$.

In the transition curves, shown in FIG. 2, three step-like features can be seen. The first, present at $\approx 8 \mathrm{~K}$, appears to be directly related to the $T_{c}$ of the bare $\mathrm{Nb}$ film. This is evidence of local regions of the bilayer film where the Er has no direct influence on the $\mathrm{Nb}$, that is, where the two materials are not coupled by the proximity effect. One possibility for this is at the Er grain boundaries or local regions around the wire-bond contacts, where the force of the contact may have disrupted the $\mathrm{Nb} / \mathrm{Er}$ interface. This interpretation is supported by the observation that there is no significant field history dependence of this step.

Some common explanations for step-like features in the transition curves can be ruled out for our system. The sputtering technique employed in this work is unlikely to create a 
significant thickness gradient. To check the uniformity of the films, a $20 \times 20 \mathrm{~mm}$ film was diced into several pieces and X-ray reflectivity was performed. A 5\% variation in thickness was observed, this variation is only slightly greater than the error in individually calculated thickness by fitting to Keissig fringes. By comparison, the sample size for transport measurements was only $3 \times 3 \mathrm{~mm}$, where uniformity in film thickness will be very high. Crystallographic inhomogeneity is a further possibility, but again unlikely. We examined a possible current (heating) dependence of the step-like features using currents ranging from $100 \mathrm{nA}$ up to $1 \mathrm{~mA}$, but found no such evidence and current induced local heating can thus be ruled out. There are no known Nb-Er alloys and in our previous reflectivity work we observed no evidence for intermixing at the interface [33], which if possible could have altered the superconducting properties of the Nb. Poor interface transparency can cause anomalous features in resistivity around the superconducting transition, as current paths change to flow preferentially through the superconductor. The formation of an oxide barrier at the interface would cause such effects, but the calculated oxidation time in our vacuum of 15 minutes is far longer than the 20 seconds between the final $\mathrm{Nb}$ and Er layer depositions. The steps are never observed in either single layer Nb films (deposited under identical growth conditions), or films of $\mathrm{Nb}$ grown in proximity to a homogeneous ferromagnet such as Co (see for example [13]).

Step-like features have been observed previously in works coupling BCS superconductors to inhomogeneous ferromagnetic textures. For example Witt et al. in helical Ho/Nb bilayers [43, 44], Yi Zhu et al. in GdN/Nb/GdN spin valves [45], and L.Y. Zhu et al. in striped domain $(\mathrm{Co} / \mathrm{Pt})_{n} / \mathrm{Nb}$ multilayers where it appears that the step shape can be modified by defining a current path parallel (no inhomogeneity - no step) or perpendicular (inhomogeneity - step) to the stripe domains [41].

While the exact origin of the step is unknown, it appears linked to the S-F proximity effect in all examples above. In this work we observe, in the upper panel of FIG. 3, that the height of the step as a fraction of the transition is field history dependent. This step height change occurs at a different field than the largest changes in $M_{r}$ and $\Delta T_{c}$. While the change in step height does not appear to be intrinsically linked to the change in $T_{c}$, it is still clearly linked to the magnetic state of the Er layer. This further supports that the origin of the step is a fundamental feature of the $\mathrm{S}-\mathrm{F}$ proximity effect, requiring theoretical description. 


\section{CONCLUSIONS}

In summary, the remanent state of Er, when proximity coupled to a $\mathrm{Nb}$ superconductor, can have a strong influence on $T_{c}$. The application of magnetic field is able to change the metastable magnetic state of the Er from a conical to fan state. This modification results in a fundamental change to the shape and temperature of the superconducting transition to zero resistivity.

We hope the observation of this unconventional effect proves fruitful for refinement of S-F theory, particularly with the lack of current description of the stepped transition observed in this (and many similar) systems.

A shift in $T_{c}$ of $140 \mathrm{mK}$ is much larger than previously observed for singlet domain wall effects and is comparable to the largest observed by the generation of the LRTC in the context of the superconducting spin valve with $3 d$ ferromagnets. This system fulfils the requirements for cryogenic memory based upon the proposed architecture of Oh et al. [3], and we offer this materials system as a candidate for future super-spintronic device application.

\section{ACKNOWLEDGMENTS}

The authors would like to thank the UK EPSRC (grant numbers: EP/J010634/1, $\mathrm{EP} / \mathrm{J} 010650 / 1, \mathrm{EP} / \mathrm{I031014/1}$ and EP/J01060X/1) for their financial support. NS acknowledges JEOL Europe and ISIS neutron and muon source for PhD funding.

The data associated with this paper are openly available from the University of Leeds data repository. https://doi.org/10.5518/142

[1] J. Linder and J. W. A. Robinson, "Superconducting spintronics," Nat. Phys. 11, 307-315 $(2015)$.

[2] M. Eschrig, "Spin-polarized supercurrents for spintronics," Phys. Today 64, 43-49 (2011).

[3] S. Oh, D. Youm, and M. R. Beasley, "A superconductive magnetoresistive memory element using controlled exchange interaction," Appl. Phys. Lett. 71, 2376-2378 (1997). 
[4] A. Potenza and C. H. Marrows, "Superconductor-ferromagnet $\mathrm{CuNi} / \mathrm{Nb} / \mathrm{CuNi}$ trilayers as superconducting spin-valve core structures," Phys. Rev. B 71, 180503 (2005).

[5] C. Bell, G. Burnell, C. W. Leung, E. J. Tarte, D.-J. Kang, and M. G. Blamire, "Controllable Josephson current through a pseudospin-valve structure," Appl. Phys. Lett. 84, 1153-1155 (2004).

[6] E. Goldobin, H. Sickinger, M. Weides, N. Ruppelt, H. Kohlstedt, R. Kleiner, and D. Koelle, "Memory cell based on a $\varphi$ josephson junction," Appl. Phys. Lett. 102, 242602 (2013).

[7] B. Baek, W. H. Rippard, S. P. Benz, S. E. Russek, and P. D. Dresselhaus, "Hybrid superconducting-magnetic memory device using competing order parameters," Nat. Commun. 5, 3888 (2014).

[8] B. M. Niedzielski, S. G. Diesch, E. C. Gingrich, Y. Wang, R. Loloee, W. P. Pratt, and N. O. Birge, "Use of Pd-Fe and Ni-Fe-Nb as soft magnetic layers in ferromagnetic Josephson junctions for nonvolatile cryogenic memory," IEEE Trans. Appl. Supercond. 24, 1-7 (2014).

[9] E. C. Gingrich, B. M. Niedzielski, J. A. Glick, Y. Wang, D. L. Miller, R. Loloee, W. P. Pratt, and N. O. Birge, "Controllable 0- $\pi$ Josephson junctions containing a ferromagnetic spin valve." Nat. Phys. 12, 564-567 (2016).

[10] P. V. Leksin, N. N. Garif'yanov, I. A. Garifullin, Ya. V. Fominov, J. Schumann, Y. Krupskaya, V. Kataev, O. G. Schmidt, and B. Büchner, "Evidence for triplet superconductivity in a superconductor-ferromagnet spin valve," Phys. Rev. Lett. 109, 057005 (2012).

[11] A. A. Jara, C. Safranski, I. N. Krivorotov, C.-T. Wu, A. N. Malmi-Kakkada, O. T. Valls, and K. Halterman, "Angular dependence of superconductivity in superconductor/spin-valve heterostructures," Phys. Rev. B 89, 184502 (2014).

[12] X. L. Wang, A. Di Bernardo, N. Banerjee, A. Wells, F. S. Bergeret, M. G. Blamire, and J. W. A. Robinson, "Giant triplet proximity effect in superconducting pseudo spin valves with engineered anisotropy," Phys. Rev. B 89, 140508 (2014).

[13] M. G. Flokstra, T. C. Cunningham, J. Kim, N. Satchell, G. Burnell, P. J. Curran, S. J. Bending, C. J. Kinane, J. F. K. Cooper, S. Langridge, A. Isidori, N. Pugach, M. Eschrig, and S. L. Lee, "Controlled suppression of superconductivity by the generation of polarized Cooper pairs in spin-valve structures," Phys. Rev. B 91, 060501 (2015).

[14] M. G. Flokstra, N. Satchell, J. Kim, G. Burnell, P. J. Curran, S. J. Bending, J. F. K. Cooper, C. J. Kinane, S. Langridge, A. Isidori, N. Pugach, M. Eschrig, H. Luetkens, A. Suter, 
T. Prokscha, and S. L. Lee, "Remotely induced magnetism in a normal metal using a superconducting spin-valve," Nat. Phys. 12, 57-61 (2016).

[15] P. V. Leksin, N. N. Garif'yanov, A. A. Kamashev, A. A. Validov, Ya. V. Fominov, J. Schumann, V. Kataev, J. Thomas, B. Büchner, and I. A. Garifullin, "Isolation of proximity-induced triplet pairing channel in a superconductor/ferromagnet spin valve," Phys. Rev. B 93, 100502 (2016).

[16] Ya. V. Fominov, A. A. Golubov, T. Yu Karminskaya, M. Yu Kupriyanov, R. G. Deminov, and L. R. Tagirov, "Superconducting triplet spin valve," JETP lett. 91, 308-313 (2010).

[17] A. Singh, S. Voltan, K. Lahabi, and J. Aarts, "Colossal proximity effect in a superconducting triplet spin valve based on the half-metallic ferromagnet $\mathrm{CrO}_{2}$," Phys. Rev. X 5, 021019 $(2015)$.

[18] S. Voltan, A. Singh, and J. Aarts, "Triplet generation and upper critical field in superconducting spin valves based on $\mathrm{CrO}_{2}, "$ Phys. Rev. B 94, 054503 (2016).

[19] N. G. Pugach, M. Safonchik, T. Champel, M. E. Zhitomirsky, E. Lähderanta, M. Eschrig, and C. Lacroix, "Superconducting spin valves controlled by spiral re-orientation in MnSi," arXiv:1702.08828 (unpublished).

[20] I. Sosnin, H. Cho, V. T. Petrashov, and A. F. Volkov, "Superconducting phase coherent electron transport in proximity conical ferromagnets," Phys. Rev. Lett. 96, 157002 (2006).

[21] J. W. A. Robinson, J. D. S. Witt, and M. G. Blamire, "Controlled injection of spin-triplet supercurrents into a strong ferromagnet," Science 329, 59-61 (2010).

[22] Y. Gu, J. W. A. Robinson, M. Bianchetti, N. A. Stelmashenko, D. Astill, F. M. Grosche, J. L. MacManus-Driscoll, and M. G. Blamire, "Magnetic state controllable critical temperature in epitaxial Ho/Nb bilayers," APL Mater. 2, 046103 (2014).

[23] Y. Gu, G. B. Halász, J. W. A. Robinson, and M. G. Blamire, "Large superconducting spin valve effect and ultrasmall exchange splitting in epitaxial rare-earth-niobium trilayers," Phys. Rev. Lett. 115, 067201 (2015),

[24] M. Habenschuss, C. Stassis, S. K. Sinha, H. W. Deckman, and F. H. Spedding, "Neutron diffraction study of the magnetic structure of erbium," Phys. Rev. B 10, 1020-1026 (1974).

[25] D. Gibbs, J. Bohr, J. D. Axe, D. E. Moncton, and K. L. D’Amico, "Magnetic structure of erbium," Phys. Rev. B 34, 8182-8185 (1986).

[26] H. Lin, M. F. Collins, T. M. Holden, and W. Wei, "Magnetic structure of erbium," Phys. 
Rev. B 45, 12873-12882 (1992).

[27] R. A. Cowley and J. Jensen, "Magnetic structures and interactions in erbium," J. Phys.: Condens. Matter 4, 9673 (1992).

[28] D. A. Jehan, D. F. McMorrow, J. A. Simpson, R. A. Cowley, P. P. Swaddling, and K. N. Clausen, "Collapsing cycloidal structures in the magnetic phase diagram of erbium," Phys. Rev. B 50, 3085-3091 (1994).

[29] B. Watson and N. Ali, "Magnetic transitions in single-crystal erbium," J. Phys.: Condens. Matter 7, 4713 (1995).

[30] B. Watson and N. Ali, "The $b$-axis magnetic phase diagram of erbium," J. Phys.: Condens. Matter 8, 1797 (1996).

[31] B. H. Frazer, J. R. Gebhardt, and N. Ali, "Magnetic phase diagrams of erbium," J. Appl. Phys. 85, 6100-6102 (1999).

[32] J. D. S. Witt, J. F. K. Cooper, N. Satchell, C. J. Kinane, P. J. Curran, S. J. Bending, S. Langridge, L. J. Heyderman, and G. Burnell, "Magnetic phases of sputter deposited thinfilm erbium," Sci. Rep. 6, 39021 (2016).

[33] N. Satchell, J. D. S. Witt, G. Burnell, P. J. Curran, C. J. Kinane, T. R. Charlton, S. Langridge, and J. F. K. Cooper, "Probing the spiral magnetic phase in $6 \mathrm{~nm}$ textured erbium using polarised neutron reflectometry," J. Phys.: Condens. Matter 29, 055801 (2017).

[34] J. Kwo, M. Hong, and S. Nakahara, "Growth of rare-earth single crystals by molecular beam epitaxy: The epitaxial relationship between hcp rare earth and bcc niobium," Appl. Phys. Lett. 49, 319-321 (1986).

[35] W. G. Moffatt, The Handbook of Binary Phase Diagrams, v. 4 (Schenectady, NY: General Electric Company, Corporate Research and Development, Technology Marketing Operation, 1981).

[36] J. Bohr, D. Gibbs, J. D. Axe, D. E. Moncton, K. L. d'Amico, C. F. Majkrzak, J. Kwo, M. Hong, C. L. Chien, and J. Jensen, "Diffraction studies of rare earth metals and superlattices," Physica B: Condens. Matter 159, 93-105 (1989).

[37] T. Löfwander, T. Champel, J. Durst, and M. Eschrig, "Interplay of magnetic and superconducting proximity effects in ferromagnet-superconductor-ferromagnet trilayers," Phys. Rev. Lett. 95, 187003 (2005).

[38] Y. V. Nazarov, "Novel circuit theory of Andreev reflection," Superlattice Microst. 25, 1221- 
1231 (1999).

[39] D. Fritsch and J. F. Annett, "Proximity effect in superconductor/conical magnet/ferromagnet heterostructures," New J. Phys. 16, 055005 (2014).

[40] A. Yu. Rusanov, M. Hesselberth, J. Aarts, and A. I. Buzdin, "Enhancement of the superconducting transition temperature in $\mathrm{Nb} /$ Permalloy bilayers by controlling the domain state of the ferromagnet," Phys. Rev. Lett. 93, 057002 (2004).

[41] L. Y. Zhu, T. Y. Chen, and C. L. Chien, "Altering the superconductor transition temperature by domain-wall arrangements in hybrid ferromagnet-superconductor structures," Phys. Rev. Lett. 101, 017004 (2008).

[42] L. Y. Zhu, Yaohua Liu, F. S. Bergeret, J. E. Pearson, S. G. E. te Velthuis, S. D. Bader, and J. S. Jiang, "Unanticipated proximity behavior in ferromagnet-superconductor heterostructures with controlled magnetic noncollinearity," Phys. Rev. Lett. 110, 177001 (2013).

[43] J. D. S. Witt, Superconductivity and Non-homogeneous Magnetism, Ph.D. thesis, Department of Materials Science and Metallurgy, University of Cambridge (2012).

[44] F. Chiodi, J. D. S. Witt, R. G. J. Smits, L. Qu, G. B. Halász, C.-T. Wu, O. T. Valls, K. Halterman, J. W. A. Robinson, and M. G. Blamire, "Supra-oscillatory critical temperature dependence of Nb-Ho bilayers," EPL 101, 37002 (2013).

[45] Y. Zhu, A. Pal, M. G. Blamire, and Z. H. Barber, "Superconducting exchange coupling between ferromagnets," Nat. Mater. 16, 195199 (2016). 